\title{
LETTER Haptically Assisting Breast Tumor Detection by Augmenting Abnormal Lump
}

\author{
Seokhee JEON ${ }^{\dagger a)}$, Member
}

SUMMARY This paper reports the use of haptic augmented reality in breast tumor palpation. In general, lumps in the breast are stiffer than surrounding tissues, allowing us to haptically detect them through selfpalpation. The goal of the study is to assist self-palpation of lumps by haptically augmenting stiffness around lumps. The key steps are to estimate non-linear stiffness of normal tissues in the offline preprocessing step, detect areas that show abnormally stiffer responses, and amplify the difference in stiffness through a haptic augmented reality interface. The performance of the system was evaluated in a user-study, demonstrating the potential of the system.

key words: haptics, augmented reality, palpation, breast tumor

\section{Introduction}

Breast self-palpation is a touch-based screening method used in an attempt to detect early breast cancer. In general, abnormal lumps in the breast are stiffer than surrounding tissues, and finding distinct softness differences of the lumps is essential in self-palpation. However, in an initial stage of tumor development, the softness difference is not very perceivable, drastically decreasing the detection rate.

Our focus in this work is to develop a medical assistance system where such softness difference is haptically amplified via a force-feedback interface, helping a user to detect the lumps. Here is an example scenario we are considering. Suppose that a woman is examining her breast by self-palpation, and an underdeveloped tumor exists in her breast. Due to its young age, the tumor's hardness might not be distinguishable, and thus she could miss detecting it in normal palpation. With our tool, however, abnormal responses of the tumor are selectively augmented, making her feel bumps around it more clearly. This eventually increases the chance that the tumor is detected.

This paper is our first attempt to realize this scenario. We introduce a new palpation assistance system where abnormal reaction force due to lumps is extracted from the response force and systematically amplified in a way that maximizes its perceptual saliency. Since the scenario involves haptic interaction with real objects, i.e., a physical breast, conventional haptic rendering framework suitable for virtual reality applications is not applicable. Instead, our system is based on an emerging haptic modeling/rendering approach called haptic augmented reality (AR), which for-

Manuscript received September 17, 2013.

Manuscript revised October 18, 2013.

${ }^{\dagger}$ The author is with the Haptics Laboratory, Kyung Hee University, Yongin-si, South Korea.

a)E-mail: Jeon@khu.ac.kr

DOI: 10.1587/transinf.E97.D.361 mulates requirements needed for interactions with real objects [1]. Thus, principles related to haptic AR are also applied to this work. Our algorithm first constructs a model representing the responses of normal physical tissue in a preprocessing step. Then, during real palpation on tissues with harder inclusion, the system calculates the force difference between normal and abnormal tissues. Based on this difference, the system provides additional reaction force when touching around the inclusion.

While there exist a few attempts on virtual reality or AR-based tumor palpation simulator for the purpose of medical training, e.g., [2]-[4] (see [5] for review), very little research has been done on assisting abnormality detection in real palpation. One example was a system by Yamamoto et al. [6]. They automatically estimated tissue stiffness and graphically displayed a colored stiffness map to assist identifying an abnormally harder tissue. However, to our knowledge, there has been no work that directly augments the haptic channel for assisting abnormal lump detection.

\section{Approach}

As a proof-of-concept study, this paper presents our initial system that only considers one-point and tool-mediated interaction. In addition, in order to focus on the core mechanism, physical tissue models are assumed homogeneous, except for embedded tumor. Moreover, instead of using a real breast, a tissue-like silicone model is used as a breast mock-up in all process to avoid safety issue.

Our approach for augmenting abnormal lump is explained using Fig. 1. As shown in the upper illustration, a user palpates a silicone breast mock-up that has no lump inside (no-tumor model) and feels pure reaction force of normal tissues at time $t$, denoted by $\mathbf{f}_{N}(t)$. Hereafter, bold letters represent $3 \mathrm{D}$ vectors. On the other hand, when there is harder lumps inside the mock-up as shown in the lower part of Fig. 1 (tumor-embedded model), the reaction force that the user feels, $\mathbf{f}_{R}(t)$, is different from $\mathbf{f}_{N}(t)$. This difference is the forces purely from the lumps, which can be written as

$$
\mathbf{f}_{L}(t)=\mathbf{f}_{R}(t)-\mathbf{f}_{N}(t) .
$$

The goal of the system is to augment or amplify the pure response of the lump, $\mathbf{f}_{L}(t)$, when palpating the tumorembedded model so as to give the user clearer perception of a harder nodule for detection. This augmentation can be realized by appropriately adding virtual force $\mathbf{f}_{D}(t)$ derived based on $\mathbf{f}_{L}(t)$ over real responses $\mathbf{f}_{R}(\mathrm{t})$ via a haptic interface. 

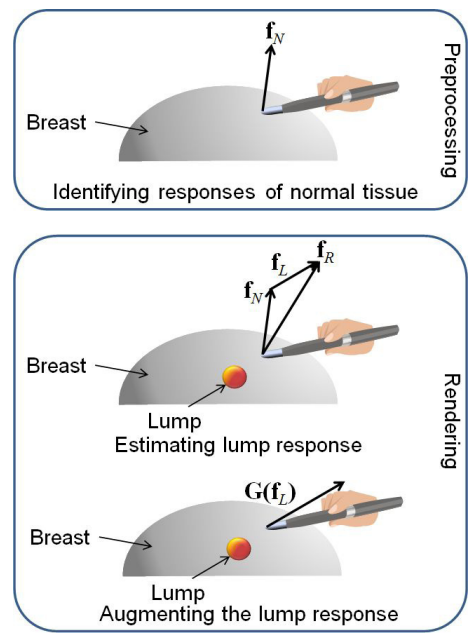

Fig. 1 Approach for lump augmentation.

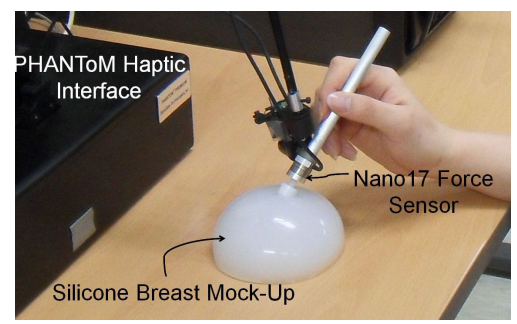

Fig. 2 Hardware configuration for lump augmentation.

Equation (1) indicates that we needs to estimate two force vectors, $\mathbf{f}_{R}(t)$ and $\mathbf{f}_{N}(t)$ to derive $\mathbf{f}_{L}(t)$. In order to measure $\mathbf{f}_{R}(t)$ in realtime, we employ a $3 \mathrm{D}$ force sensor at the tip of the tool as shown in Fig. 2. $\mathbf{f}_{N}(t)$ can be estimated by identifying a contact dynamics model of a no-tumor model in a preprocessing step as shown in the upper part of Fig. 1. Detailed explanation about the identification is reported in Sect. 3 .

After estimating $\mathbf{f}_{L}(t)$, we need to determine a function that calculates the amplification force $\mathbf{f}_{D}(t)$ depending on $\mathbf{f}_{L}(t)$. This function is denoted by $\mathbf{f}_{D}(t)=G\left(\mathbf{f}_{L}(t)\right.$ ) (see the lower part of Fig. 1). This function should be carefully chosen while taking into account a perceptual performance of the amplification as well as the performance of the haptic interface. Section 4 explains this process.

$\mathbf{f}_{D}(t)$ determines the force that the haptic interface has to exert to the user. To physically generate the force, we use our haptic AR hardware already introduced in [7]. The hardware consists of an impedance-type haptic device (PHANToM premium 1.5; SensAble Technologies) and a customdesigned interaction tool attached to it. The tool is instrumented with 3D force/torque sensors (Nano17; ATI Industrial Automation, Inc.) as shown in Fig. 2.

\section{Response Identification}

A preprocessing step is introduced in order to estimate tissue responses, $\mathbf{f}_{N}(t)$, to be used in rendering. In this step, we identify a contact dynamics model of the no-tumor model using data gathered via active palpation of this model with the tool in Fig. 2, As a contact dynamics model, the HuntCrossley model [8] is used. The model is based on Hertz elastic theory and has been applied to model 1D viscoelastic contact dynamics of deformable objects such as human tissue [6]. In the model, the response force under a certain displacement, $x(t)$, and velocity, $\dot{x}(t)$, is determined by

$$
f(t)=k\{x(t)\}^{m}+b\{x(t)\}^{m} \dot{x}(t),
$$

where $k$ and $b$ are stiffness and damping constants, and $m$ is a constant exponent (usually $1 \leq m \leq 2$ ).

The identification procedure is adapted from our previous publication [7]. In summary, while repeatedly pressing and releasing the no-tumor model with the tool, we record data triples consisting of the displacement, velocity, and reaction force along surface normal at the initial contact point, denoted by $\left(x_{N}, \dot{x}_{N}, f_{N}\right)$. The data are passed to a recursive least-squares algorithm for iterative Hunt-Crossley model parameter estimation. This model is denoted as $H_{N}(x, \dot{x})$ and will be used to determine $\mathbf{f}_{N}(t)$ in the next section.

\section{Algorithm for Lumps Augmentation}

During rendering, a user makes a contact with the mockup (tumor-embedded model) with the tool, which initiates augmentation. To detect the moment of this contact, we examine abrupt rise of the force sensor reading as already introduced in our previous publication [7].

After a contact is detected, the user palpates the mockup with the tool, making it deform. The deformation generates reaction force to the user's hand, $\mathbf{f}_{R}(t)$, which is measured via the force sensor. This force is different from the response of the no-tumor model, $\mathbf{f}_{N}(t)$, and the difference becomes a pure tumor force, $\mathbf{f}_{L}(t)$, as aforementioned in Eq. (1).

$\mathbf{f}_{N}(t)$ is estimated as follows. In fact, $H_{N}(x, \dot{x})$ derived in Sect. 3 only represents 1D response at a certain point of the no-tumor model. Based on $H_{N}(x, \dot{x})$ and under the homogeneity assumption, 3D responses at arbitrary location is estimated using an algorithm previously published in [7]. In summary, the direction of $\mathbf{f}_{N}(t)$ is determined by a vector connecting from current haptic tool position to the initial contact point, whereas the magnitude of the vector is estimated based on linearly normalized $H_{N}(x, \dot{x})$ and the distance between the tool tip and the initial contact point.

After estimating $\mathbf{f}_{N}(t), \mathbf{f}_{N}(t)$ and $\mathbf{f}_{R}(t)$ is passed into Eq. (1), determining $\mathbf{f}_{L}(t)$. Now the core part of the augmentation is how to determine the amplification force $\mathbf{f}_{D}(t)$ for a better detectability. The augmentation is realized by providing an additional force directed by $\mathbf{f}_{L}(t)$ such that

$$
\mathbf{f}_{D}(t)=G\left\{\left|\mathbf{f}_{L}(t)\right|\right\} \frac{\mathbf{f}_{L}(t)}{\left|\mathbf{f}_{L}(t)\right|},
$$

where $G$ determines the magnitude of amplification based on the magnitude of $\mathbf{f}_{L}(t)$. 
$G$ should be chosen carefully to maximize the detectability. Larger amplification gain may increase the detectability, but there is a certain limit bounded by the maximum exertable force of the interface. In this paper, we suggest two options for $G$ considering this limit.

The first method linearly relates the magnitude of $\mathbf{f}_{L}(t)$ with the gain such that

$$
G_{1}\left\{\left|\mathbf{f}_{L}(t)\right|\right\}= \begin{cases}n\left|\mathbf{f}_{L}(t)\right|, & \text { if } n\left|\mathbf{f}_{L}(t)\right|<f_{\text {max }} \\ f_{\text {max }}, & \text { otherwise, }\end{cases}
$$

where $n$ is a linear gain, and $f_{\text {max }}$ is a maximum exertable force of the interface. The second method put bigger gain on smaller difference such that

$$
G_{2}\left\{\left|\mathbf{f}_{L}(t)\right|\right\}=f_{\max }\left\{-\frac{p}{\left|\mathbf{f}_{L}(t)\right|+p}+1\right\},
$$

where $p$ decides the degree of gain increment along smaller to larger difference. Finally, $\mathbf{f}_{D}(t)$ is determined by Eq. (3) and is sent to the haptic interface.

\section{Implementation Results}

To build no-tumor and tumor-embedded mock-up, we casted a mixture of Ecoflex 0030 (SmoothOn Inc.) and silicone thinner into a breast-shaped mold $(55-\mathrm{mm}$ radius half sphere). A no-tumor sample had uniform viscoelasticity, and its linear stiffness measured at $10 \mathrm{~mm}$ displacement was $0.21 \mathrm{~N} / \mathrm{mm}$. A tumor-embedded sample was also prepared, which had an identical material to the no-tumor sample, except for harder tumors of $6.5-\mathrm{mm}$ radius inserted at $25 \mathrm{~mm}$ below the surface. The stiffness of the tumor measured at $3 \mathrm{~mm}$ indentation was $0.41 \mathrm{~N} / \mathrm{mm}$. Then, the responses of the no-tumor sample was identified as described in Sect. 3. An example of measured and estimated responses (and additionally $\mathbf{f}_{R}$ for reference) are shown in Fig. 3 .

Then, the tumor-embedded model was palpated, and pure tumor responses were estimated using the identified model. The pure tumor response was then fed into $G$, and final force commands were sent to the haptic interface. For the actual force rendering, we used the openHaptics library supplied from the PHANToM manufacturer. The update rate of the haptic control loop was kept $1 \mathrm{kHz}$ for stable rendering. Figure 4 shows an example of an estimated tumor response $\left(\mathbf{f}_{L}\right)$, and corresponding augmentation forces $\left(\mathbf{f}_{D}\right)$

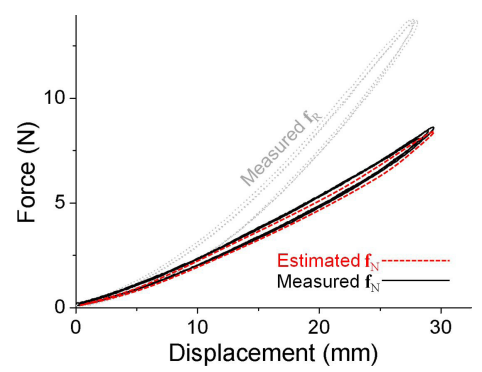

Fig. 3 Estimated and measured displacement vs. reaction force curves of the no-tumor model. derived from the two amplification methods.

Overall, the stability of amplification force rendering depends on the parameters in $G$. In case of $G_{1}, n$ higher than 2.3 showed unstable rendering, which seems due to commanded forces frequently reaching to the maximum force limit of the PHANToM (as shown in the figure for the case of $n=3$ ). For $G_{2}$, on the other hand, $p$ lower than 0.2 resulted in unstable rendering. Again, lower $p$ easily makes commanded force value very close to the limit and causes rapid changes in force command, decreasing the stability.

\section{User Study}

\subsection{Methods}

Twelve subjects (10 males and 2 females; 22-35 years old) participated in the experiment. All of them were righthanded and haven't experienced haptic interfaces before. None of them were aware of the purpose of the experiment.

In the experiment, the participants palpated breast mock-ups using the system. The same no-tumor and tumorembedded models as in Sect. 5 were used in the experiment. The size and stiffness of the tumor in the tumor-embedded model were carefully determined in a pilot test. They are chosen so that the existence of the tumor is barely detectable through careful palpation for considerable time.

In the experiment, the participants were instructed to find a tumor while palpating the breast mock-ups. There were four experimental conditions. In the first condition, a no-tumor model without any physical or virtual tumor was palpated, designated as NO_TUM. The second condition, NO_AUG provided a tumor-embedded model without virtual augmentation. In the third and fourth conditions, AUG1 and AUG2, a tumor-embedded model was palpated with virtual augmentation, but first (linear function) and second (rational function) augmentation methods were used, respectively. The parameter, $n$ of the linear method was set to 2.2 , and $p$ of the rational function method was 0.6 , which were carefully selected to maximize the amplification while still preserving stability of rendering. With these four conditions, the within-subject design was employed. The participants were informed that models may not have a tumor.

The experiment began with 10 min training session where palpation was practiced. Both no-tumor and tumor-

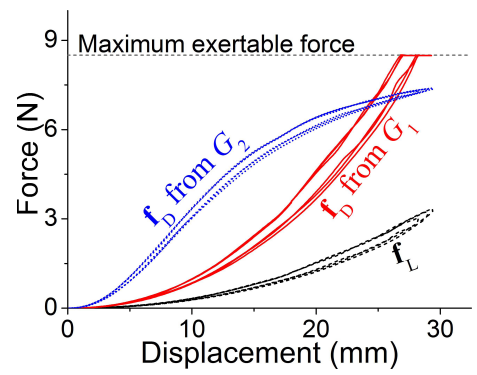

Fig. 4 Estimated pure tumor response and amplification forces from $G_{1}$ and $G_{2}$. In this example, $n$ in $G_{1}$ was 3 , and $p$ of $G_{2}$ was 0.5 . 
embedded models were given to the participant, allowing them to experience the difference. In the main session, the participant palpated each condition 10 times, resulting in 40 trials. Any order effect was minimized by balancing the conditions across the participants using a Latin Square.

In each trial, the participants were instructed to find the tumor as soon as possible through palpation and to press a space bar when a tumor was found. The time taken to find a tumor was recorded for each trial. Maximum 1 minute was given for a trial. If a tumor was not found within this time period, it was considered that the participant failed to find a tumor. The number of the failure was also recorded. The experimenter randomly rotated the breast mock-up to change tumor positions. The whole experiment lasted for 40 minute. Auditory cues were blocked by earplugs worn by the participants. Visual cues were also blocked since the appearances of the two mock-ups were identical to each other.

\subsection{Results and Discussion}

The average detection times and detection rates for the four conditions are shown in Fig. 5 with standard errors as error bars. The NO_TUMOR condition shows the worst results in both criteria. However, the rate is not $0 \%$, and the time did not reach $60 \mathrm{sec}$, which indicates that the participants sometimes declared the existence of a tumor even when there is no tumor. Comparing to the NO_TUMOR condition, the participants spent less time in the NO_AUG condition, which is expected. When the NO_AUG condition is compared with the augmentation conditions (AUG1 and AUG2), one can clearly see that the augmentation helped with detecting a tumor. The detection rates of the AUG1 and AUG 2 conditions are all 100\%, whereas the tumor could not be detected in $13 \%$ of trials in NO_AUG condition. In addition, compared to the NO_AUG condition, about 50\% less time was taken for detection in the AUG1 condition,

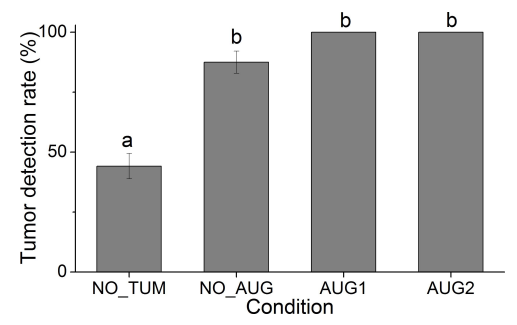

(a) Tumor detection rate.

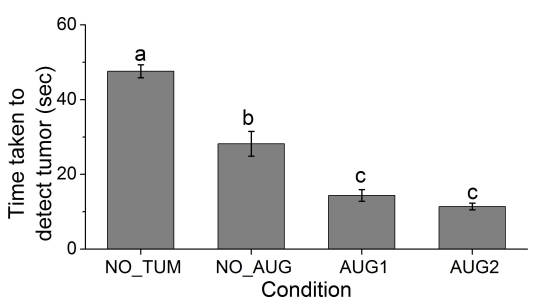

(b) Tumor detection time.

Fig.5 Average tumor detection rate and time for the four conditions. Statistically same groups are marked by the same letters. while the AUG2 condition showed $60 \%$ improvement.

One-way within-subject ANOVA revealed that the experimental condition had a statistically significant effect in both measurements $\left(F_{3,33}=56.88, p<0.0001\right.$ for the detection rate and $F_{3,33}=62.77, p<0.0001$ for the detection time). A post-hoc comparison (the Student-NewmanKeuls test) was also conducted. For the detection rate, NO_AUG, AUG1, and AUG2 had statistically same means while NO_TUM condition was not. For the detection time, all conditions had statistically different means, except for the AUG1 and AUG2 conditions.

The experimental results demonstrated that the augmentation helps a user with detecting tumor. The detection time is significantly reduced when the augmentation is applied. The detection rate was also improved, while it was not statistically significant. Participants' suggestions to the system were also collected after the main session. They all reported that the system was very helpful to find a tumor, which specifically shows the effectiveness of the system.

Nevertheless, the participants complained that the feeling of the tumor was not very realistic; tumor did not feel as a normal static nodule that they have experienced so far, but it felt as if it is actively moving. We speculate that this happened in the AUG2 condition, where bigger reaction force was rendered at small displacement, being felt active. However, this is actually expected result since even this unrealistic response can be a cue for tumor detection. Other comments complained about the way of palpation; tool-mediated palpation is not a natural way of palpation. We will tackle this issue in future work by introducing a tool providing a bare-hand interaction.

The augmentation of lumps inherently amplifies measuring and modeling errors as well. This may increase a false alarm rate, i.e., declaring a tumor in a no-tumor model, particularly when $G_{2}$ function is used for the amplification. Although our previous work has proven that the errors themselves are perceptually small enough in spite of the material homogeneity assumption [7], the effect is unclear if they are amplified. In order to reveal the effect of the amplification on false detection, a small informal experiment was conducted with four new participants. The experimental procedure was identical to that described in Sect.6.1, except for experimental conditions. Three conditions are tested: 1) the NO_TUM condition (for comparison), 2) palpating a no-tumor model while $G_{2}$ amplification is applied (main condition), 3) the AUG2 condition (to prevent guessing). Figure 6 shows the result on tumor detection rate. In spite that no statistical meaning can be drawn due to small number of participant, one can see that the increment of the false alarm rate due to the amplification is marginal (see the difference between the first and the second condition in the figure). From this observation, we speculate that the effect of error amplification is not severe and does not significantly reduce the applicability of the system. Final note on this informal experiment is that the false alarm rate of the NO_TUM condition (35\%) does not coincide with that in the main experiment (44.17\%). This is due to the differ- 


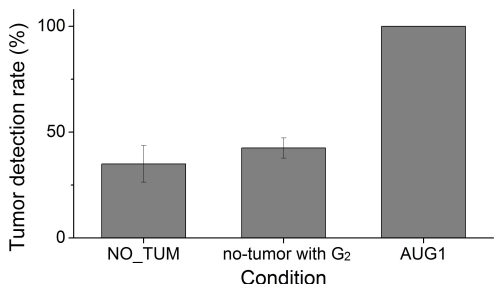

Fig. 6 False detection rate.

ence in experimental conditions; only one condition had a tumor in this experiment while there were three in the main experiment, and thus participants are more likely to deny the existence of a tumor.

\section{Conclusions}

In this paper, we developed a haptic AR system that assists in finding tumors in a palpation scenario by extracting and augmenting the responses of the tumor. All required algorithms as well as hardware were developed. The performance of the system in terms of detection rate and time was confirmed in a user experiment.

Several future directions are found. One tangible improvement is multi-contact-points interaction. Another future work is the bare-hand interaction, which needs a miniaturized force sensor and new tool design.

\section{Acknwoledgments}

This work was supported by the MSIP Korea, under the
ITRC program (NIPA-2013- H0301-13-4006) supervised by the NIPA (National IT Industry Promotion Agency) and NRF through ERC 2011- 0030075.

\section{References}

[1] S. Jeon and S. Choi, "Haptic augmented reality: Taxonomy and an example of stiffness modulation," Presence: Teleoperators and Virtual Environments, vol.18, no.5, pp.387-408, 2009.

[2] M. Cheng, W. Marinovic, M. Watson, S. Ourselin, J. Passenger, H. De Visser, O. Salvado, and S. Riek, "Abdominal palpation haptic device for colonoscopy simulation using pneumatic control," IEEE Trans. Haptics, vol.5, no.2, pp.97-108, 2012.

[3] S. Ullrich and T. Kuhlen, "Haptic palpation for medical simulation in virtual environments," IEEE Trans. Vis. Comput. Graph., vol.18, no.4, pp.617-625, 2012.

[4] M. Alhalabi, V. Daniulaitis, H. Kawasaki, and T. Hori, "Medical training simulation for palpation of subsurface tumor using HIRO," Proc. EuroHaptics, pp.623-624, 2005.

[5] S. Jeon, S. Choi, and M. Harders, "Rendering virtual tumors in real tissue mock-ups using haptic augmented reality," IEEE Trans. Haptics, vol.5, no.1, pp.77-84, 2012.

[6] T. Yamamoto, B. Vagvolgyi, K. Balaji, L.L. Whitcomb, and A.M. Okamura, "Tissue property estimation and graphical display for teleoperated robot-assisted surgery," Proc. IEEE International Conference on Robotics and Automation, pp.3117-3123, 2009.

[7] S. Jeon and S. Choi, "Real stiffness augmentation for haptic augmented reality," Presence: Teleoperators and Virtual Environment, vol.20, no.4, pp.337-370, 2011.

[8] K. Hunt and F. Crossley, "Coefficient of restitution interpreted as damping in vibroimpact," ASME J. Applied Mechanics, vol.42, pp.440-445, 1975. 